\title{
Collective deceleration: Toward a compact beam dump
}

\author{
H.-C. Wu, ${ }^{1}$ T. Tajima, ${ }^{1,2}$ D. Habs, ${ }^{1,2}$ A. W. Chao, ${ }^{3}$ and J. Meyer-ter-Vehn ${ }^{1}$ \\ ${ }^{1}$ Max-Planck-Institut für Quantenoptik, D-85748 Garching, Germany \\ ${ }^{2}$ Fakultät für Physik, Ludwig-Maximilians-Universität München, D-85748 Garching, Germany \\ ${ }^{3}$ SLAC National Accelerator Center, Stanford University, Stanford, California 94309, USA
}

(Received 10 December 2009; published 5 October 2010)

\begin{abstract}
With the increasing development of laser electron accelerators, electron energies beyond a GeV have been reached and higher values are expected in the near future. A conventional beam dump based on ionization or radiation loss mechanisms is cumbersome and costly, not to mention the radiological hazards. We revisit the stopping power theory of high-energy charged particles in matter and discuss the associated problem of beam dumping from the point of view of collective deceleration. The collective stopping length in an ionized gas can be several orders of magnitude shorter than that described by the Bethe-Bloch formulas and associated with multiple electromagnetic cascades in solids. At the same time, the tenuous density of the gas makes the radioactivation negligible. Such a compact beam dump without radioactivation works well for short and dense bunches, as they are typically generated from a laser wakefield accelerator. In addition, the nonuniform transverse wakefield can induce microbunching of the electron bunch by betatron oscillation. The microstructure could serve as a prebunched source for coherent radiation or feeding a free electron laser.
\end{abstract}

DOI: 10.1103/PhysRevSTAB.13.101303

PACS numbers: 29.27.Bd, 41.75.Ht, 52.40.Mj

\section{INTRODUCTION}

As accelerators of particles (electrons and ions) acquire higher energies and fluence, the issue of the radiological safety for the operation of such accelerators is increasingly important. At the end of each particle accelerator, one needs to decelerate particles into a safe energy region, so that there is little radioactivation induced by the highenergy particles in the environment, and no radiation hazard for the laboratory staff. One has to be cautious concerning the design of the beam dump and the radiation shielding. The beam dump may contain both high- $Z$ and low- $Z$ materials and have a thick concrete surrounding. The whole safety system must be validated and monitored. Such a safety issue becomes cumbersome and expensive with increasing particle energy, especially for tabletop laser wakefield accelerators.

In this paper, we suggest to use collective forces in matter (in particular in plasmas) for deceleration. We shall find that short and dense bunches of electrons and other particles such as positrons are amenable under appropriate conditions to be stopped over distances many orders of magnitude shorter than in a conventional beam dump with solid matter. The required plasma density is low so that hazardous radioactivation due to individual nuclear collisions can be reduced significantly. This compact and safe beam dump becomes more important for particle energies beyond a $\mathrm{GeV}$, a regime in which secondary particles, like muons, can be generated which are heavy and need a longer distance for stopping in condensed matter.

In order to make the accelerator and its associated beam dump system compact and safe, we can marshal collective interaction that can far surpass in magnitude over the conventional individual forces, provided that proper conditions are met. In the present article we focus on deceleration. However, we emphasize that there can be a general consideration of overall utilization of collective force for the purpose of beam dynamics that allows us to make the system far more compact than conventional methods. Using electric (and sometimes magnetic) fields of collective origin in the plasma, one can focus electron [1] and ion [2] beams by means of so-called plasma lens. Together with the plasma beam dump considered below, we can call these efforts collective plasma optics.

The paper is organized as follows. To compare with the conventional beam dump, in Sec. II, we present a review on the stopping power in matter based on the Bohr-FermiBethe-Bloch theory. In Sec. III, we give the stopping power of the collective deceleration for dense and ultrashort electron bunches and compare it with the classic stopping power. PIC simulations describing the collective deceleration of an electron bunch in underdense plasma are given in Sec. IV. We find that deceleration in a uniform plasma becomes ineffective after a certain distance. When that happens, a periodic-structured plasma is proposed to further decelerate the electrons. Moreover, a microbunching structure with a period much smaller than the plasma wavelength develops during the deceleration process. An analytic description of the microbunching process is given, and its potential applications are discussed. The final Sec. V draws a conclusion.

\section{BOHR-FERMI-BETHE-BLOCH THEORY}

The conventional beam dump is designed based on the understanding of the Bohr-Fermi-Bethe-Bloch classic the- 
ory on the stopping power in matter [3-9]. A classic formula given first by Bohr [3] in 1913, and later modified by Bethe [5] and Bloch [6] into a quantum-mechanical formula, which is now universally called the Bethe-Bloch formula $[7,8]$ of the stopping power (for relativistic electrons in condensed matter), reads

$$
-(d E / d x)_{I}=\left(F / \beta^{2}\right)\left[\ln \left(2 m_{e} \gamma^{2} v^{2} / I\right)-\beta^{2}\right],
$$

where $E$ is the electron kinetic energy, $F=$ $4 \pi e^{4} n_{e, m} / m_{e} c^{2}=e^{2} k_{p e, m}^{2}, n_{e, m}$ is the electron density in the stopping material, $k_{p e, m}=\omega_{p e, m} / c$ is the plasma wave number, and $\beta=v / c$ is the normalized electron velocity. The electron energy is absorbed by excitation or ionization of bound electrons in the atoms of the stopping material, and $I$ represents a specific average of the excitation and ionization potentials in the atom. The dominant mechanism of Bethe-Bloch stopping power is the charged particle interaction with electrons in matter resulting in ionization. The logarithm term within the bracket is around 20 for a broad range of parameters.

In Ichimaru's treatment of the Bethe-Bloch formula in plasma [9], the stopping power is clearly attributed to the part due to the binary collisions and that to the long-ranged collective interaction, where the beam particle is treated as a single test particle. In other words, $-(d E / d x)_{P}=$ $-(d E / d x)_{\text {ind }}-(d E / d x)_{\text {coll }}$, where the first term

$$
-(d E / d x)_{\text {ind }}=\left(F / \beta^{2}\right) \ln \left(m_{e} v^{2} / e^{2} k_{D}\right)
$$

arises from individual-particle collisions with the characteristic wave number limited by $m_{e} v^{2} / e^{2}$ and down to the Debye wave number $k_{D}$, while the second term

$$
-(d E / d x)_{\text {coll }}=\left(F / \beta^{2}\right) \ln \left(k_{D} v / \omega_{p e}\right)
$$

is the contribution from collectively excited plasma waves with wave number $k<k_{D}$. Interestingly, the contributions to the plasma stopping power by individual binary collisions and by plasma collective oscillations can be combined together to yield $-(d E / d x)_{P}=\left(F / \beta^{2}\right) \times$ $\ln \left(m_{e} v^{3} / e^{2} \omega_{p e}\right)$.

For relativistic electrons, the other important energy loss from individual collisions is due to bremsstrahlung radiation [4] of electrons. In place of Eq. (1) or Eq. (2), the stopping power due to radiation loss reads

$$
-(d E / d x)_{R}=F(Z / 137 \pi)(\gamma-1) \ln \left(183 Z^{-1 / 3}\right),
$$

where $Z$ is atomic number. The approximate ratio of the two losses is $(d E / d x)_{R} /(d E / d x)_{\text {ind }}=E Z / 1600 m_{e} c^{2}$ [5]. Thus, radiation loss is dominant for electrons at higher energy, e.g. $E>100 \mathrm{MeV}$ for $Z=10$.

\section{COLLECTIVE DECELERATION}

The use of collective fields of plasma for particle acceleration was first suggested by Veksler [10] (deceleration in the present context). It may allow interaction to be en- hanced above and beyond the single particle level [Eq. (2)] and the linear level of collective fields [Eq. (3)] both for stopping and for acceleration. With the development of powerful lasers and high-current relativistic electron bunches, the new method of laser (or plasma) wakefield acceleration has been proposed to accelerate electrons by exploiting collective plasma fields, such as by laser $[11,12]$ and by electron beams [13].

The wakefield amplitude, when driven at resonance of the plasma wave (i.e. at the eigenfrequency of collective plasma oscillations) by the strong ponderomotive force of these drivers, becomes highly nonlinear and grows beyond the applicability of perturbative theory. Laser wakefield acceleration in this highly nonlinear regime leads to bubblelike wakes from which all electrons are blown out [14]. It may be only characterized by the nonperturbative limit of the wave-breaking field [11] $m_{e} c \omega_{p e} / e$ and that driven by the electron bunch $[15,16] m_{e} c \omega_{p e}\left(n_{b} / n_{e}\right) / e$, as the wakefield in this limit has a cusp singularity [17], where $n_{b}$ and $n_{e}$ are the electron bunch and plasma densities, respectively. This collective stopping power for wakefield deceleration of the electron bunch is large:

$$
-(d E / d x)_{\text {coll-wave break }}=m_{e} c \omega_{p e}\left(n_{b} / n_{e}\right) .
$$

Equation (5) is exact for the resonant excitation of a wakefield with bunch length $\sigma_{L} / \lambda_{p e} \approx 0.5$, transverse size $\sigma_{T} / \lambda_{p e} \geq 0.3$, and modest density ratio $n_{b} / n_{e}<10$ [16]. For a long beam $\sigma_{L} / \lambda_{p e} \gg 1$, the stopping power decreases exponentially with the factor $k_{p e} \sigma_{L} \times$ $\exp \left(-k_{p e}^{2} \sigma_{L}^{2} / 2\right)$. For a narrow beam $\sigma_{T} / \lambda_{p e} \ll 1$, the stopping power decreases with the factor $k_{p e}^{2} \sigma_{T}^{2}$. Note that, in linear theory, the wakefield excited by a single test charge in the long wavelength limit of the plasma collective field is given by Eq. (3), to be compared with the nonlinear wakefield stopping power given by Eq. (5).

In order to optimally generate this nonperturbative plasma wakefield, a dense electron bunch with $n_{b} \geq n_{e}$ is required. To avoid self-injection of plasma electrons, however, we need $n_{b} \leq n_{e}$. As a satisfactory compromise, we choose to stay around $n_{b} / n_{e} \sim 1$. Thus, the ratio of collective deceleration in plasma and the Bethe-Bloch stopping power in condensed matter is

$R=\frac{(d E / d x)_{\text {coll-wave break }}}{(d E / d x)_{\text {ind }}} \approx \frac{m_{e} c \omega_{p e} \beta^{2}}{F \Lambda}=\frac{n_{e}}{n_{e, m}} \frac{\lambda_{p e}}{r_{0}} \frac{\beta^{2}}{2 \pi \Lambda}$,

where $\lambda_{p e}$ is the plasma wavelength of the background plasma with density $n_{e}, r_{0}$ is the classical electron radius, and $\Lambda$ is the logarithm term. On the other hand, according to Eq. (2), the ratio of the stopping power due to the individual interaction (short-range) $(d E / d x)_{\text {ind }}$ in plasma to that in a conventional solid dump, $(d E / d x)_{\text {ind }}$, is $n_{e} / n_{e, m}$ and several orders of magnitude less than unity. This contributes to the significant reduction of the amount of nu- 
clear activation due to individual nuclear collisions in the plasma dump.

For a typical example with $n_{e}=10^{19} \mathrm{~cm}^{-3}, n_{e, m}=3 \times$ $10^{23} \mathrm{~cm}^{-3}$, and $\lambda_{p e}=10 \mu \mathrm{m}$, we have $R \approx 1000$, i.e., the deceleration distance in the underdense plasma is 3 orders of magnitude smaller than the stopping in condensed matter. Because of this [Eq. (6)], the collective deceleration by a tenuous gas (which will be quickly turned into ionized plasma by the electric field of the impinging bunch) is capable of stopping beams over distances many orders of magnitude shorter $(1 / R)$ than in the conventional solid beam dump. Nevertheless, compared to solids, the radioactivating hazard is reduced by many orders of magnitude $\left(n_{e} / n_{e, m}\right)$ due to the tenuity of the gas and its consequent scarcity of binary collision. We simultaneously accomplish the enhancement of the stopping power and the reduction of the binary collisions both by many orders of magnitude.

In relativistic regimes beyond $\mathrm{GeV}$, in addition to the multiple cascades of electrons, bremsstrahlung photons by the radiation loss generate muon pairs by means of photonuclear reactions. The muon fluence is highly peaked in the forward direction. Additional material is needed for stopping them [18]. Because of the heavier mass, $m_{\mu}=$ $206.8 m_{e}$, muons are more penetrative than electrons. The stopping mechanism for muons is the ionization loss. Usually, several meters of high- $Z$ metals are needed to stop the muons. Its stopping power is $-(d E / d x)_{\text {ind, } \mu}=$ $-(d E / d x)_{\text {ind }}\left(m_{e} / m_{u}\right)$, where $-(d E / d x)_{\text {ind }}$ is the electron stopping power given by Eq. (1). Compared with the collective deceleration in plasma, one has

$$
R_{\mu}=\frac{(d E / d x)_{\text {coll-wave break }}}{(d E / d x)_{\text {ind, }, \mu}}=R \frac{m_{\mu}}{m_{e}},
$$

where $R$ is given by Eq. (6). In the example of the last paragraph, this ratio takes the value as large as $R_{\mu} \approx 2 \times$ $10^{5}$. Thus, for energies beyond a $\mathrm{GeV}$ and taking muons into account, the effective stopping power of collective deceleration in a plasma is even more pronounced when compared with the Bethe-Bloch stopping power in condensed matter.

It is noted that collective energy loss due to various beam plasma instabilities has been discussed in a number of papers, see e.g. [19-22]. Weibel instability may lead to beam filamentation, and subsequent filament coalescence has been identified as a very effective mechanism of collective deceleration [19]. Beam-excited Langmuir turbulence has been described as a way to stop collectively electron bunches right in the center of inertial fusion targets for fast ignition [20]. Enhanced resistivity may result from wave-particle interactions due to ion Bernstein mode instability in current-carrying plasma [21]. In these cases, the beam density needs to be comparable to the plasma density, just as for the collective deceleration discussed in the present paper, but the temporal and/or spatial scales on which these instabilities de- velop are different. They are ineffective for the electron bunches of a few micrometer diameter considered here. The collective energy loss of an attosecond electron pulse in overdense plasma has been discussed in Ref. [22]. Here, the main difference is that the present paper is focused on a practical design of a beam dump for laser electron accelerators. It is demonstrated explicitly in terms of 2D-PIC simulations in the next section.

\section{PARTICLE SIMULATION}

\section{A. Collective deceleration and saturation}

Here we examine the feasibility of a beam dump using collective deceleration in a tenuous plasma. For this we carry out a series of two-dimensional (2D) particle-in-cell (PIC) simulations [23], representing the central results of this paper. As we shall see, our beam dump is highly effective for short and dense beams. Beams from laser wakefield accelerators (LWFA) are in fact very short and dense. Therefore, we take typical parameters of a bunch from LWFA [24]. The electron bunch has a total charge 50-100 pC, and a spherical distribution with a diameter of $3 \mu \mathrm{m}$. The bunch density is $n_{b} \approx 2.2-5 \times 10^{19} \mathrm{~cm}^{-3}$. A beam divergence of $\theta=1 \mathrm{mrad}$ is used to calculate the bunch size after a certain vacuum drift. As shown in Ref. [25], such an ultrashort bunch mainly experiences transverse expansion. The energy spread is taken as $1 \%$. The electron bunch is injected into the plasma and propagates from left to right along the $x$ axis. The simulation box has a size of $10 \lambda_{p e} \times 10 \lambda_{p e}$, and it moves with the speed of light.

Figure 1 shows the decrease of the total bunch energy as a function of the propagation distance in a nonstructured uniform plasma of density $n_{e}=n_{b} / 5 \approx 1.1 \times 10^{19} \mathrm{~cm}^{-3}$. In this case, the normalized transverse size and the longitudinal length of the bunch are $\sigma_{T} / \lambda_{p e}=\sigma_{L} / \lambda_{p e}=0.3$.

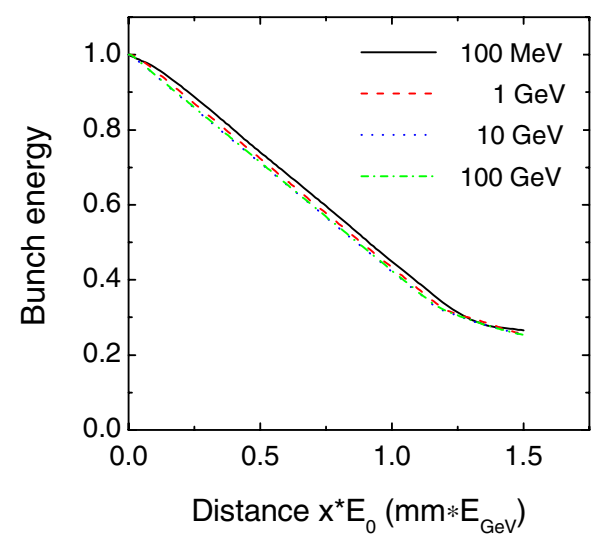

FIG. 1. Collective deceleration and its saturation. The relative decrease of total bunch energy is given versus scaled propagating distance for different initial bunch energies $E_{0}: 100 \mathrm{MeV}, 1 \mathrm{GeV}$, $10 \mathrm{GeV}$, and $100 \mathrm{GeV}$. The plasma density is $n_{e}=n_{b} / 5 \approx 1.1 \times$ $10^{19} \mathrm{~cm}^{-3}$. 
The initial particle energy $E_{0}$ is varied from $100 \mathrm{MeV}$ to $100 \mathrm{GeV}$.

It is seen that the bunch energy drops linearly with distance. Apparently, the stopping power is independent of the initial particle energy in this region, until some kind of saturation sets in after a saturation length $L_{s}$. The deceleration distance is proportional to the bunch energy and is in fact determined by the stopping power given in Eq. (5), i.e., the wakefield amplitude is independent of the bunch energy. For a bunch of $1 \mathrm{GeV}$ electrons initially, $75 \%$ of the energy is deposited in a plasma column $1.5 \mathrm{~mm}$ long. Beyond the saturation length, the electron deceleration becomes much slower and almost vanishes.

In order to better understand the saturation mechanism, Fig. 2 provides the distribution of energy vs the $x$ position of all electrons in the bunch around the distance $L_{s}$. Here we take the initial bunch energy of $500 \mathrm{MeV}$ and a plasma density of $n_{e}=2 n_{b} \approx 4.4 \times 10^{19} \mathrm{~cm}^{-3}$. In this case, the normalized bunch sizes are $\sigma_{T} / \lambda_{p e}=\sigma_{L} / \lambda_{p e}=0.6$. Figure 2(a) shows that the bunch tail is effectively decelerated and, in Fig. 2(b), some tail electrons are completely stopped toward zero velocity and lag behind the main bunch. Then, these lagging electrons are trapped in the acceleration phase of the wakefield and regain energy, as shown in Fig. 2(c). In fact, the bunch is already split into three parts: electrons at the bunch front that feel almost no deceleration field, electrons in the middle still decelerating, and electrons at the tail already regaining energy. It is at this point when the overall deceleration saturates. The total energy evolution is shown in Fig. 3(b) for the case of the uniform plasma. The remaining energy after saturation is about $25 \%$.

\section{B. Beam dump with structured plasmas}

In order to circumvent saturation in a uniform plasma and to further decelerate the bunch, we suggest to employ a structured plasma for phase mismatch control [26], as it is shown in Figs. 3(a) and 4(a). Just before the moment when some tail electrons are completely stopped, we replace the uniform plasma by some periodic plasma slabs with vac- uum gaps or periodic thin foils inserted in the background uniform plasma. It is expected that those electrons which approach to come to rest can be retained around the vacuum gap or the foil, so that they are not trapped in the plasma for renewed acceleration.

In the case with vacuum gap, we set the thickness of the plasma slab equal to the vacuum gap. Figure 3(b) shows that, after a deceleration in the $1.15 \mathrm{~mm}$ long uniform plasma, the bunch energy can indeed further decelerate when introducing plasma slabs with either $L_{P} / \lambda_{p e}=2,5$, or 10 periods. After a distance of $3 \mathrm{~mm}, 90 \%$ bunch energy is absorbed. Further deceleration is possible if more plasma slabs are used.

As expected, Fig. 3(c) shows that only a low-energy electron tail is left after the main bunch. Most of these low-energy electrons have a kinetic energy smaller than $5 \mathrm{MeV}$. Electrons with energy less than $10 \mathrm{MeV}$ are safer because they do not lead to radioactivation. Only the bunch head cannot effectively be decelerated, because the wakefield is weak on the bunch head.

To check the robustness of deceleration in the structured plasma, we consider an electron bunch after a $1 \mathrm{~cm}$ vacuum drift, in which the transverse size becomes $\sigma_{T}=10 \mu \mathrm{m}$, while the longitudinal length of $\sigma_{L}=3 \mu \mathrm{m}$ does not change. The bunch density is $n_{b} \approx 2 \times 10^{18} \mathrm{~cm}^{-3}$. We vary the plasma density from $n_{e} / n_{b}=1$ to $n_{e} / n_{b}=80$. The corresponding normalized bunch length varies from $\sigma_{L} / \lambda_{p e}=0.13$ to $\sigma_{L} / \lambda_{p e}=1.2$ and the bunch width from $\sigma_{T} / \lambda_{p e}=0.4$ to $\sigma_{T} / \lambda_{p e}=3.8$. For the case of a uniform plasma shown in Fig. 3(d), the rate of energy loss decreases for $\sigma_{L} / \lambda_{p e}>1$. This is because the bunch is too long for the wakefield, having a length of $\sim \lambda_{p e}$. In this case, the bunch tail is always reaccelerated [15]. The optimal wakefield is generated for $\sigma_{L} / \lambda_{p e}=0.5$. In other words, the proposed beam dump is effective when

$$
\sigma_{L} / \lambda_{p e}<1 \text {. }
$$

This implies that (i) the shorter the bunch is, the higher the plasma density can be taken and the shorter becomes the stopping length [see Eq. (5)], and that (ii) the denser
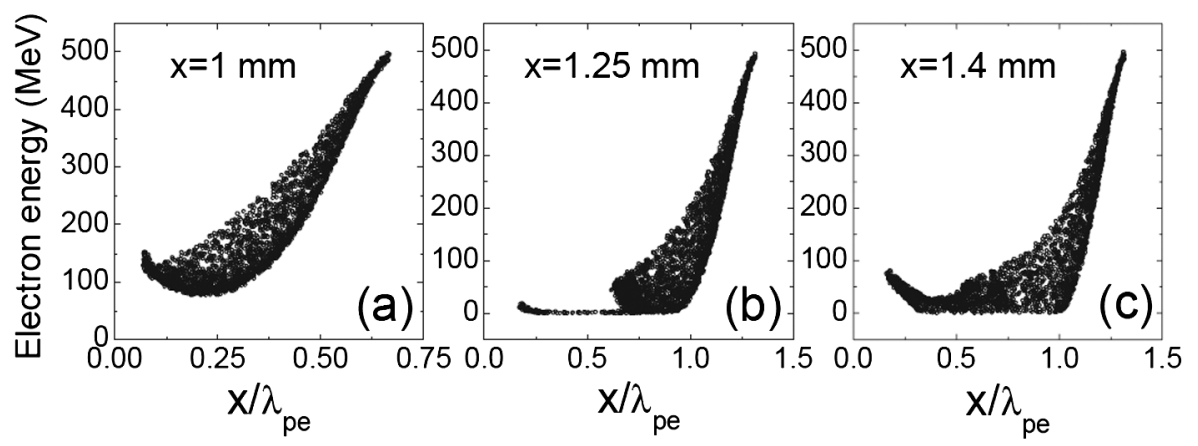

FIG. 2. Electron energy distribution during collective deceleration in nonstructured plasma. Energies are given versus longitudinal position ( $x$ in units of plasma wavelength) at different propagating distances: (a) $x=1 \mathrm{~mm}$, (b) $x=1.25 \mathrm{~mm}$, and (c) $x=1.4 \mathrm{~mm}$. The plasma density is $n_{e}=2 n_{b} \approx 4.4 \times 10^{19} \mathrm{~cm}^{-3}$, and the initial energy of bunch electrons is $500 \mathrm{MeV}$. 

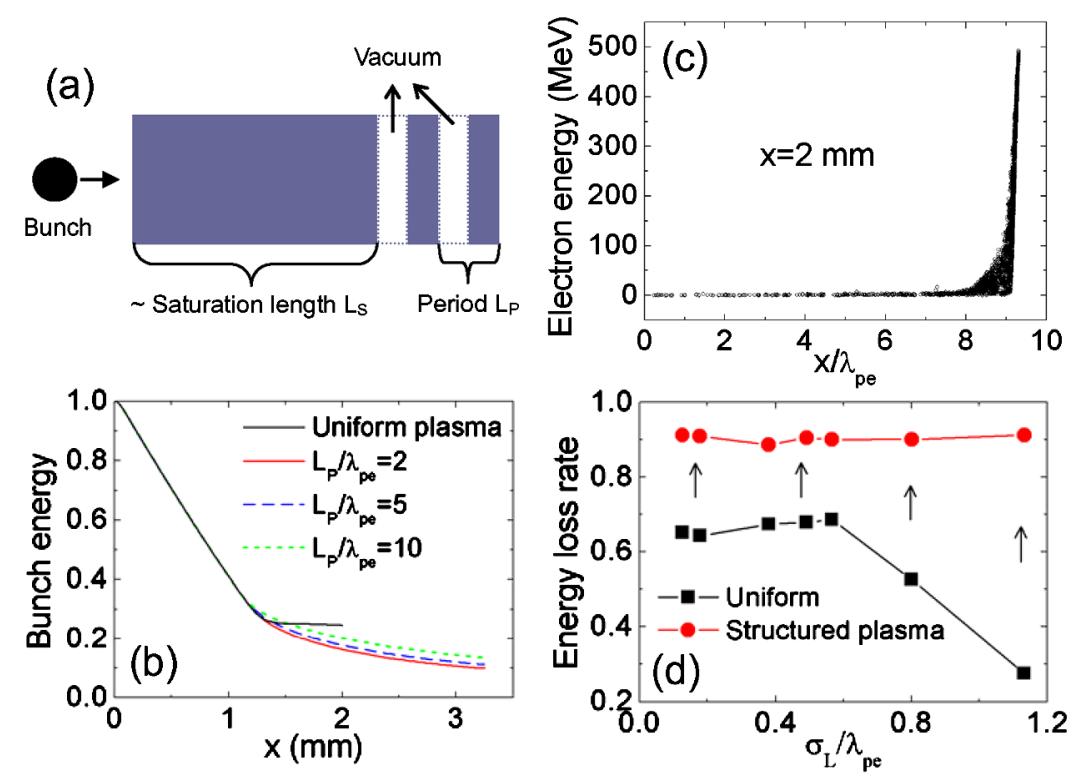

FIG. 3. Suggested beam dump. (a) The proposed structured plasma consisting of a stopping layer with thickness $L_{s}$ and periodic plasma slabs separated by a vacuum gap. $L_{p}$ is the period of the structured plasma. In each period, the plasma slab length is equal to the vacuum length. (b) Evolution of bunch energy for both uniform plasma and structured plasma with $L_{P} / \lambda_{p e}=2,5$, and 10. (c) Electron energy vs $x$ position at $x=2 \mathrm{~mm}$ for the case of $L_{P} / \lambda_{p e}=2$. (d) Improvement of energy loss rate due to the structured plasma for different plasma densities plotted in terms of $\sigma_{L} / \lambda_{p e}$, keeping bunch sizes $\sigma_{T}=10 \mu \mathrm{m}$ and $\sigma_{L}=3 \mu \mathrm{m}$ constant.

bunch is more effective [also see Eq. (5)]. For the structured plasma case, the rate of energy loss can reach to $90 \%$. The results in Fig. 3 show that the structured plasma with vacuum gaps is applicable and robust for a broad range of bunch or plasma parameters.

Figure 4(a) shows the alternative scheme of a structured plasma with periodic thin foils dipping into the uniform plasma. This scheme may be easier to implement in experiments. With this method, as illustrated in Fig. 3, we can also improve the deceleration efficiency after deceleration in the $1.15 \mathrm{~mm}$ long uniform plasma. The length and separation of foils are $0.1 \lambda_{p e}$ and $L_{P}=1 \lambda_{p e}$, respectively. The foil density is 100 times of the background plasma, i.e. $n_{\text {foil }} \approx 4.4 \times 10^{21} \mathrm{~cm}^{-3}$, which is a typical density of solid aerogel. As shown in Fig. 4(b), after a distance of $3 \mathrm{~mm}$,
$85 \%$ of the bunch energy is absorbed. Figure 4(c) shows a low-energy electron tail behind the main bunch. Most of these low-energy electrons have a kinetic energy smaller than $10 \mathrm{MeV}$. Further deceleration is possible for more plasma/foil periods and optimized parameters of foil density and thickness.

In addition, we have also examined the case of positron beam deceleration. We have found from our simulations that positron bunches can be decelerated in the same way as electron bunches.

\section{Microbunching of the decelerated bunch}

Simulations also show that the electron bunch can develop a substructure of microbunches during collective deceleration. This is illustrated in Fig. 5 for the beam and
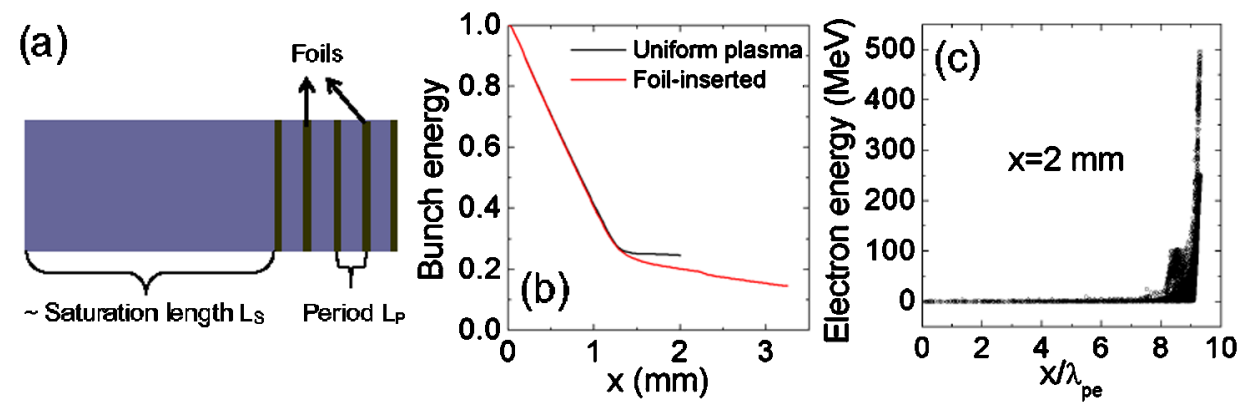

FIG. 4. Suggested beam dump. (a) The structured plasma with periodic thin foils inserted. $L_{p}$ is the separation of the neighboring foils. (b) Bunch energy evolution for both uniform plasma and the structured plasma with $L_{P} / \lambda_{p e}=1$, foil length $0.1 \lambda_{p e}$, and density $n_{\text {foil }}=100 n_{e}$. (c) Electron energy vs $x$ position at $x=2 \mathrm{~mm}$. 

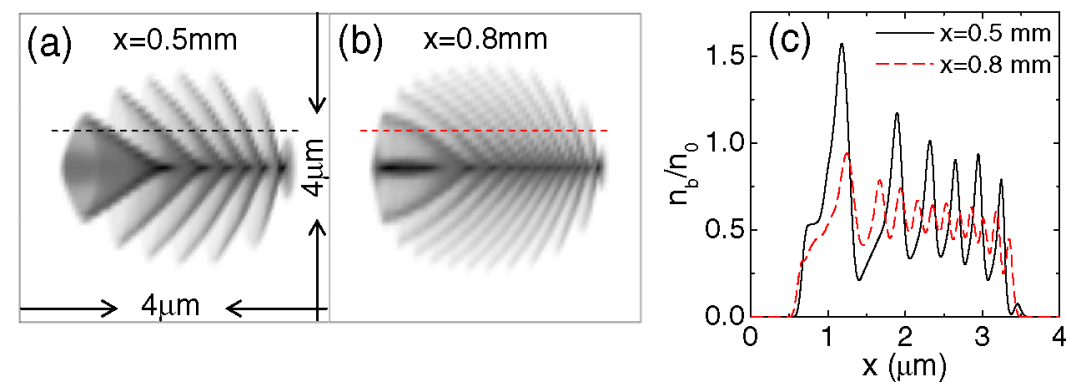

FIG. 5. Microbunching during deceleration. Snapshots of bunch density for the propagation distances (a) $x=0.5 \mathrm{~mm}$ and (b) $x=0.8 \mathrm{~mm}$. (c) Display of bunch density distributions along the dashed lines in (a) and (b). Simulation parameters are the same as in Fig. 2.

plasma parameters given in Fig. 2. The electron bunch carries out betatron oscillations in the transverse direction. The modulation period of the microbunch structure decreases with the propagation distance. The reason for the microbunch generation is the nonuniform radial wakefield along the longitudinal direction within the bunch. The electron bunch can be considered as a set of infinitely thin sheets along the $x$ direction. If the radial field is uniform along the $x$ direction, the radius of each sheet oscillates synchronously with the same betatron frequency. For our case, the wakefield is weak towards the bunch head and is strong toward its tail. The different sheets therefore have different betatron frequencies and the resulting nonsynchronous oscillations lead to the bunch envelope modulation.

Since the beam deceleration works near the blow-out regime, we assume that electron bunch blows out all the plasma electrons, and leaves a positive ion column. The transverse electrostatic field of the ion column is $2 \pi n_{e} e r$, and the electron motion in this transverse field satisfies $d p_{T} / d t=-2 \pi n_{e} e^{2} r$. For relativistic electrons, we have $\gamma_{T}=1 / \sqrt{1-v_{T}^{2}} \ll \gamma$, and the equation of electron motion becomes

$$
d^{2} r / d t^{2}=-\Omega_{b}^{2} r,
$$

where $\Omega_{b}=\omega_{p e} / \sqrt{2 \gamma}$ is the betatron frequency. Since a relativistic electron has $t \simeq x / c$, the motion equation can be rewritten as $d^{2} r / d x^{2}+\left(\Omega_{b}^{2} / c^{2}\right) r=0$, where $x$ is the electron propagation coordinate.

If we neglect the effects of emittance, space charge, and self-magnetic field of the electron bunch, we can obtain from Eq. (9) the envelope equation of the bunch $[27,28]$ as

$$
\left[\frac{\partial^{2}}{\partial x^{2}}+\frac{\Omega_{b}^{2}(\xi)}{c^{2}}\right] \sigma_{T}(x, \xi)=0,
$$

where $\xi=x-c t$ is the comoving coordinate of the bunch. We consider the front part of electron bunch within $\xi \in$ $\left[-\sigma_{L}^{\prime}, 0\right]$, where $\sigma_{L}^{\prime} \leq \sigma_{L}$. We assume the radial field increases linearly from the bunch head $\xi=0$ to the position $\xi=-\sigma_{L}^{\prime}$, so one has

$$
\Omega_{b}(\xi)=\Omega_{b 0}\left(1+\xi / \sigma_{L}^{\prime}\right),
$$

where $\Omega_{b 0}=\omega_{p e} / \sqrt{2 \gamma}$ is the maximum betatron frequency. The solution of Eq. (10) is

$$
\sigma_{T}(x, \xi)=\sigma_{T}(0, \xi)\left|\cos \left[\Omega_{b 0}\left(1+\xi / \sigma_{L}^{\prime}\right) x / c\right]\right| .
$$

The modulation period of the bunch envelope as a function of $\xi$ is

$$
\eta=\frac{\pi c \sigma_{L}^{\prime}}{\Omega_{b 0} x}=\sqrt{\frac{\gamma}{2}} \frac{\sigma_{L}^{\prime}}{x} \lambda_{p e},
$$

which decreases with the propagation distance $x$. For the case of Fig. 5, we find $\sigma_{L}^{\prime} \approx 0.5 \lambda_{p e}$. Substituting $\gamma=$ 1000 and $\lambda_{p e}=5 \mu \mathrm{m}$, we obtain $\eta_{0.5 \mathrm{~mm}} \approx 0.56 \mu \mathrm{m}$ and $\eta_{0.8 \mathrm{~mm}} \approx 0.35 \mu \mathrm{m}$, which agree with the median in Fig. 5(c). The chirped structure in Fig. 5(c) is due to the nonlinear wakefield rising within the bunch.

Such a microstructured electron bunch can potentially be a source for coherent radiation or can feed a free electron laser, and its generation requires only a short plasma insertion. Of course, additional investigations on optimum microbunch generation are needed for a practical application in this direction. We notice that there remains some chirp in the period of the microbunches. Since we understand the reason for this in the nonlinear chirp of the betatron frequency, we can utilize this or control it. It may also be possible to use this new microbunching mechanism to generate trains of zeptosecond electron pulses from an attosecond bunch, as described in Ref. [22]. Such zeptosecond pulse trains can be used as diagnostics tool for resolving ultrafast phenomena in atomic and nuclear physics.

\section{CONCLUSION}

In conclusion we have suggested to make use of collective deceleration in plasma as a beam dump mechanism for electron accelerators. This new method provides a beam dump capability that is some $3-5$ orders of magnitude more efficient than a conventional beam dump. It reduces the radioactivation hazard by many orders of magnitude. It 
could dramatically decrease the cost of the beam dump and relax the severity of the radiation shielding. The conditions necessary for effective collective deceleration call for short and dense beams. These conditions ideally match the beam characteristics of laser electron accelerators. Thus, this technique could eventually serve the development of a high-energy laser particle accelerator system in quite a unique fashion. Together with other collective plasma optics, we may design a compact accelerator and its associated systems including the collective decelerator presented here. Furthermore, in addition to LWFA, future accelerators with short bunches such as ILC [29] and CLIC [30] may find the current beam decelerator useful.

In principle, the energies from the decelerated beams deposited in the form of organized plasma wakefields, unlike the heat energy in a conventional dump, may be recovered into electricity [31]. This may be returned to drive the accelerator, saving energy. In the single particle dynamics of the Bethe-Bloch conventional beam dump, the energy of the beam electrons is converted by individual collisions into other electrons (and ions), emitting bremsstrahlung radiation and inducing other radiative processes. These converted energies are random individual-particle motions, which eventually become heat and produce a large amount of entropy. More importantly, they produce radioactivities, because collision energies exceed the threshold of the radioactivating process. This is particularly severe when the electron energy exceeds $\mathrm{GeV}$ and muons are generated. We then have to cope with the neutron generation. On the contrary, the present collective deceleration beam dump converts most of the beam electron energy into the collective plasma oscillations (i.e. electron collective motions) with little increase of entropy. The present method can stand alone without energy recovery. In this case we need to cool the plasma to remove the heat. However, because the energy of the plasma electrons is much less than that of the beam electrons, the collisions do not give rise to excessive radioactivation.

When we opt for the energy recovery, this cooling problem is mitigated. The collective oscillations of plasma involve a large electric field (on the order of $m \omega_{p} c / e$ ) in a plasma channel. Any electric (or magnetic) circuit such as a metallic loop in the plasma (not contacting the beam, but immersed in the plasma) picks up these large electromagnetic oscillations. Note that the plasma collective oscillations involve collective and coherent electric currents. These currents go through the pickup circuit. This circuit may be connected to the external circuitry, such as another wave duct or other electric circuit, from which one can extract electric energies. In this way we should recover a major fraction of energies unspent in the plasma by turning them into electricity rather than heat. We also envision that this method of energy recovery should be applicable to recovering the remaining wakefield energies in plasma capillaries, after (or while) beam loading from wakefields occurs in LWFA capillaries to reduce the heat problem in the accelerator section.

Finally, the microbunching mechanism clarified in this paper may provide a new method for seeding of free electron lasers and generation of ultrashort (zeptosecond) bunch trains. More details on this point deserve separate research.

\section{ACKNOWLEDGMENTS}

This work was supported by Deutsche Forschungsgemeinschaft through the DFG-Cluster of Excellence Munich-Centre for Advanced Photonics (MAP) and Transregio TR18. Hui-Chun Wu acknowledges support from the Alexander von Humboldt Foundation.

[1] P. Chen, Part. Accel. 20, 171 (1987).

[2] T. Toncian, M. Borghesi, J. Fuchs, E. d'Humieres, P. Antici, P. Audebert, E. Brambrink, C. A. Cecchetti, A. Pipahl, L. Romagnani, and O. Willi, Science 312, 410 (2006).

[3] N. Bohr, Philos. Mag. 25, 10 (1913); K. Dan. Vidensk. Selsk. Mat.-Fys. Medd. 18, 8 (1948).

[4] E. Fermi, Z. Phys. 29, 315 (1924).

[5] H. A. Bethe, Ann. Phys. (Leipzig) 397, 325 (1930); Z. Phys. 76, 293 (1932).

[6] F. Bloch, Ann. Phys. (Leipzig) 408, 285 (1933); Z. Phys. 81, 363 (1933).

[7] E. Segre, Nuclei and Particles: An Introduction to Nuclear and Subnuclear Physics (Benjamin, Reading, MA, 1965).

[8] C. Amsler et al., Phys. Lett. B 667, 1 (2008); A. Ferrari et al., CERN-2005-10, INFN/TC_05/11, SLAC-R-773, 2005; G. Battistoni et al., in Proceedings of the Hadronic Shower Simulation Workshop 2006, Fermilab, edited by M. Albrow and R. Raja, AIP Conf. Proc. No. 896 (AIP, New York, 2007), p. 31.

[9] S. Ichimaru, Basic Principles of Plasma Physics: A Statistical Approach (Benjamin, Reading, MA, 1973).

[10] V. I. Veksler, in Proceedings of the CERN Symposium on High Energy Accelerators and Pion Physics, Geneva, Switzerland (CERN, Geneva, 1956), Vol. 1, p. 68.

[11] T. Tajima and J. M. Dawson, Phys. Rev. Lett. 43, 267 (1979).

[12] W. P. Leemans, B. Nagler, A. J. Gonsalves, Cs. Toth, K. Nakamura, C. G. R. Geddes, E. Esarey, C. B. Schroeder, and S. M. Hooker, Nature Phys. 2, 696 (2006).

[13] P. Chen, J. M. Dawson, R. W. Huff, and T. Katsouleas, Phys. Rev. Lett. 54, 693 (1985).

[14] A. Pukhov and J. Meyer-ter-Vehn, Appl. Phys. B 74, 355 (2002).

[15] E. Esarey, P. Sprangle, J. Krall, and A. Ting, IEEE Trans. Plasma Sci. 24, 252 (1996).

[16] W. Lu, C. Huang, M. M. Zhou, W. B. Mori, and T. Katsouleas, Phys. Plasmas 12, 063101 (2005).

[17] G. Mourou, T. Tajima, and S. Bulanov, Rev. Mod. Phys. 78, 309 (2006).

[18] Radiological Safety Aspects of the Operation of Electron Accelerators (IAEA, Vienna, 1979). 
[19] M. Honda, J. Meyer-ter-Vehn, and A. Pukhov, Phys. Rev. Lett. 85, 2128 (2000).

[20] V. M. Malkin and N. J. Fisch, Phys. Rev. Lett. 89, 125004 (2002).

[21] D. R. Welch, T. C. Genoni, and D. V. Rose, B. V. Oliver, K. D. Hahn, and E. Schamiloglu, Phys. Plasmas 13, 103106 (2006).

[22] A. Ogata, T. Kondoh, K. Norizawa, J. Yang, and Y. Yoshida, Jpn. J. Appl. Phys. 48, 056002 (2009).

[23] H.-C. Wu, Z.-M. Sheng, and J. Zhang, Phys. Rev. E 77, 046405 (2008).

[24] S. Karsch, J. Osterhoff, A. Popp, T. P. Rowlands-Rees, Zs Major, M. Fuchs, B. Marx, R. Hörlein, K. Schmid, L. Veisz, S. Becker, U. Schramm, B. Hidding, G. Pretzler, D.
Habs, F. Grüner, F. Krausz, and S. M. Hooker, New J. Phys. 9, 415 (2007).

[25] F. J. Grüner, C. B. Schroeder, A. R. Maier, S. Becker, and J. M. Mikhailova, Phys. Rev. ST Accel. Beams 12, 020701 (2009).

[26] T. Tajima, Laser Part. Beams 3, 351 (1985).

[27] J. J. Su, T. Katsouleas, J. M. Dawson, and R. Fedele, Phys. Rev. A 41, 3321 (1990).

[28] S. V. Bulanov, G. Mourou, and T. Tajima, Phys. Lett. A 372, 4813 (2008).

[29] http://www.linearcollider.org/about/Publications/ Reference-Design-Report.

[30] H. Braun et al., CERN, Report No. CLIC-764, 2008.

[31] T. Tajima and A. Chao, patent applied (2008). 